\title{
Does cognitive flexibility change the nature of the relationship between intolerance of uncertainty and psychological symptoms during the COVID-19 outbreak in Turkey?
}

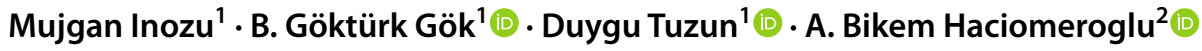 \\ Accepted: 28 October 2021 \\ ๑) The Author(s), under exclusive licence to Springer Science+Business Media, LLC, part of Springer Nature 2021
}

\begin{abstract}
The COVID-19 pandemic has significantly affected the mental health of the general population worldwide. The study aimed to determine the associations of the intolerance of uncertainty (IU) and cognitive flexibility (CF) with a variety of psychological symptoms and to examine the impact of IU on psychological symptoms through the moderating role of CF. The Brief Symptom Inventory, Intolerance of Uncertainty Scale-12, and Cognitive Control and Flexibility Questionnaire were applied to a sample of 3004 adults living in Turkey. The results of the analysis indicated that CF moderated the effect of IU on psychology symptoms. The slope analysis revealed a weakening association between IU and psychological symptoms with the introduction of a high CF level introduced to the model. The findings highlighted the importance of IU as a potential risk factor for developing psychological symptoms while $\mathrm{CF}$ appears as a potential protective factor during the COVID-19 pandemic.
\end{abstract}

Keywords COVID-19 · Psychological symptoms $\cdot$ Cognitive flexibility $\cdot$ Intolerance of uncertainty $\cdot$ Moderation

\section{Introduction}

Ever since the COVID-19 outbreak was declared a pandemic by the World Health Organization in March 2020, the world has been battling with the aftermath of this unprecedented incident (WHO, 2020). As of this moment, there are nearly 240 million cases and 4.8 million deaths globally. In Turkey, 7,154,070 people have contracted the virus since the declaration of the first case on March 10, 2020, and the numbers keep increasing by around 28,000 cases daily. The total number of deaths is 62,054 and the number continues to rise (WHO, 2021). The accelerating numbers indicate that even after a year, COVID-19 is a global and national threat. Daily curfews, physical distancing, temporary closure of public places (i.e., restaurants, shops, etc.), and mandatory face masks are some of the current restrictions implemented

Mujgan Inozu

mujganinozu@hacettepe.edu.tr

1 Department of Psychology, Hacettepe University, 06800 Ankara, Çankaya, Turkey

2 Department of Psychology, Ankara Haci Bayram Veli University, 06560 Ankara, Turkey by the Turkish government for protection. These created financial, social, and psychological burdens for individuals. People lost their jobs, closed down their businesses, failed to access essential services (i.e., health), experienced a decrease in social contact or support due to isolation, and faced much more adverse consequences during the process (Moreno et al., 2020; Stanton et al., 2020).

There is growing evidence showing the altering effect of COVID-19 on psychological wellbeing. In China, the immediate assessment of the psychological effects of COVID-19 indicated that more than half of the participants had experienced a significant degree of depression, anxiety, or stress (Wang et al., 2020). In other studies, post-traumatic stress symptoms such as re-experiencing and sleep disturbances were documented (Liu et al., 2020) as well as phobias and obsessive or psychotic symptoms (Tian et al., 2020). Results from different countries were consistent in portraying the psychological impact of COVID-19. Combined evidence from Italy, Spain, India, Australia, Germany, and the UK demonstrated an increased prevalence of depression (15\% to $48 \%$ ), anxiety (6\% to $51 \%$ ), stress, or post-traumatic stress (7\% to 82\%) (Li \& Wang, 2020; Mazza et al., 2020; Petzold et al., 2020; Varshney et al., 2020; Xiong et al., 2020). 
The examination of factors related to the escalation of psychological symptoms has become important for researchers. People with certain socio-demographic characteristics were found to be more susceptible to developing more severe symptoms. In brief, women, people younger than 18 years of age, and people older than 50 years of age experienced more psychological symptoms. Moreover, living alone, being unemployed or working in healthcare, having a lower income level, having lower education, contracting the virus, or having a relative who had contracted the virus were associated with increased rates of experiencing adverse psychological symptoms (Cao et al., 2020; Huang \& Zhao, 2021; Liu et al., 2020; Mazza et al., 2020; Tian et al., 2020; Varshney et al., 2020; Wang et al., 2020).

With reference to previous research, particular psychological factors in addition to socio-demographic characteristics were investigated. A study examined stress from negative consequences of COVID-19 (i.e., canceling plans in school or work, being infected), acute stress disorder symptoms, and possible associated psychological factors. Their results revealed the mediating role of resilience, social support, and adaptive ways of coping in the relationship between COVID-19-related stress and acute stress symptoms. All three mediators were identified as protective factors that alleviate stress-related symptoms (Ye et al., 2020). Another study aimed to define the risk and protective factors that are related to the mental outcomes of the pandemic and found that people who had high neuroticism, agreeableness, and conscientiousness were more prone to experience severe psychological symptoms while being more resilient and having better coping skills were reversely associated with psychological symptoms. Thus, the former trait characteristics were identified as risk factors while the latter were identified as protective factors (Fernández et al., 2020).

The intolerance of uncertainty (IU) can be defined as the tendency to react negatively to uncertainty (Buhr \& Dugas, 2002). People with high IU perceive uncertainty as threatening, interpret IU as a possible adverse experience, and respond with avoidance or impaired functioning (Dugas et al., 2004). Previous studies have primarily revealed a unique relationship between IU, worry, and generalized anxiety disorder, followed by studies on the significant association between IU and depression, panic disorder, and obsessive-compulsive disorder (Dugas \& Ladouceur, 2000; Gorka et al., 2014; Lind \& Boschen, 2009; Yook et al., 2010). Considering its relationship with multiple psychological disorders, IU is now recognized as a transdiagnostic maintenance factor that can be specifically targeted in treatment (McEvoy \& Mahoney, 2012). Focusing on repetitive thoughts about uncertainty (i.e., uncertainty is dangerous), challenging the repetitive thoughts, and understanding how IU negatively affects behaviors (i.e., avoidance) and feelings (i.e., constant worry) have resulted in a significant decrease in the symptoms (McEvoy \& Erceg-Hurn, 2016).

The unclear nature of the COVID-19 pandemic elevated the levels of anxiety, depression, and stress symptoms and numerous studies have confirmed the influence of IU on this elevation (Mazza et al., 2020; Wang et al., 2020). In a study, the escalated levels of insomnia were associated with IU. People who already face problems when dealing with uncertainty developed worry and stress, which, in turn, have created cognitive arousal that disturbed normal sleeping patterns (Voitsidis et al., 2020a). Furthermore, uncertainty about the duration of the pandemic contributed to the increased levels of depression and anxiety in individuals with eating disorders (Schlegl et al., 2020). The results from a study examining the effect of IU and loneliness in older adults have shown that IU regulates the level of loneliness. Especially uncertainty about the duration of the lockdowns or not knowing the required duration to maintain social distancing from relatives or friends were discussed in relation to their contribution to the exacerbation of the feelings of being lonely (Parlapani et al., 2020). Further research has demonstrated that individuals with higher IU also had lower psychological well-being during the pandemic. Moreover, COVID-19-related rumination and fear mediated this association (Satici et al., 2020). The relationship between the levels of social isolation and anxiety was moderated by IU, thus showing the association of higher IU levels with higher levels of reported anxiety (Smith et al., 2020). In light of repeated evidence from previous research, IU can be asserted as a potential risk factor for the development and maintenance of psychological symptoms that have emerged during the COVID-19 pandemic.

Cognitive flexibility (CF) is described as the ability to alter self cognitively to adapt to constantly changing situations (Dennis \& Vander Wal, 2010). CF consists of discarding insignificant information and directing focus on resources that are more of service (Diamond, 2013). Naturally, when the point of view is changeable, individuals can re-evaluate adverse experiences, stressful situations, or negative feelings and be less prone to damages (Cheng et al., 2014). Conversely, the lower levels of CF were associated with rumination, depression, and anxiety (Dennis \& Vander Wal, 2010). CF coincides with another construct called psychological flexibility, which also means adjusting behaviors in response to changes in the environment with the intention being personal wellbeing (Dawson \& Golijani-Moghaddam, 2020). CF, on the other hand, is associated more with executive functioning skills such as attention and inhibition. However, a broad review of the two constructs have shown that they overlap on many levels, but CF might be a component of psychological flexibility (Whiting et al., 2017).

The number of studies examining the role of CF in psychological wellbeing during the pandemic is relatively 
small. A study conducted in Italy aimed to investigate the prevalence of insomnia and associated psychological factors. The results demonstrated a clear link between lower $\mathrm{CF}$ and more severe sleep problems. The researchers argued that the perceived stress of the pandemic caused increased levels of insomnia and the increase was associated with lower CF (Bacaro et al., 2020). Further research reported that individuals with lower psychological flexibility experienced more COVID-19-related depression, anxiety, or worry during the pandemic while higher psychological flexibility was associated with mental wellbeing as it allows choosing appropriate coping mechanisms to better adapt to a novel situation (Dawson \& Golijani-Moghaddam, 2020; Pakenham et al., 2020). In conclusion, findings have supported the importance of $\mathrm{CF}$ as a protective factor in adapting to the unique living conditions caused by the COVID-19 pandemic by preventing the development or worsening of psychological symptoms and distress.

Preceding studies provided consistent evidence on how the COVID-19 pandemic poses a serious threat to psychological wellbeing (Liu et al., 2020; Petzold et al., 2020). The restrictions and the uncontrollability of the situation are examples of constantly changing circumstances that demand flexibility. Furthermore, not knowing when the restrictions and the pandemic will end put an extra strain on individuals despite their already low tolerance of uncertainty (Parlapani et al., 2020). In such unprecedented times, IU may remain as a risk factor in the process and negatively affect mental health while CF can act as a protective factor in adapting to the existing changes and reducing the risk of developing psychological symptoms. Thus, the present study aimed to investigate the association between IU, CF, and psychological symptoms and to examine the interactions between the three variables with a proposed moderation model depicted in Fig. 1. We hypothesized that there would be a significant positive relationship between IU and psychological symptoms. Moreover, $\mathrm{CF}$ would show a significant negative relationship with IU and psychological symptoms. Finally, CF would moderate the relationship between IU and psychological symptoms.

\section{Methodology}

\section{Participants}

The study was conducted with a cross-sectional approach where quantitative data were obtained using self-report questionnaires. The sample of the present study was composed of 3867 participants. Inclusion criteria comprised having internet access, being over 18 years of age, giving informed consent, residing in Turkey, and being literate in Turkish. According to the exclusion criteria, 31 individuals who did not reside in Turkey, 6 individuals under the age of 18, 19 individuals who did not approve the informed consent form, and 807 individuals who did not complete the questionnaires were excluded from the study. The final sample comprised 3004 participants. We have reached participants from 76 provinces of Turkey. In the sample, $71.2 \%$ of the participants were female, $28.4 \%$ were male, and $0.4 \%$ did not want to specify their gender. The mean age of the participants was $34.4(\mathrm{SD}=14.60)$ years. The majority of the participants were university graduates (undergraduate, graduate, and $\mathrm{PhD}$ ) with $67 \%$ $(\mathrm{N}=2014)$ while the remaining $33 \%(\mathrm{~N}=990)$ were either high school, secondary school, or primary school graduates. The marital status of the participants was as follows: $38.3 \%(\mathrm{~N}=1151)$ of the participants stated being married, $56.2 \%(\mathrm{~N}=1689)$ stated being single, and 5.5\% $(\mathrm{N}=164)$ stated being divorced or widowed.

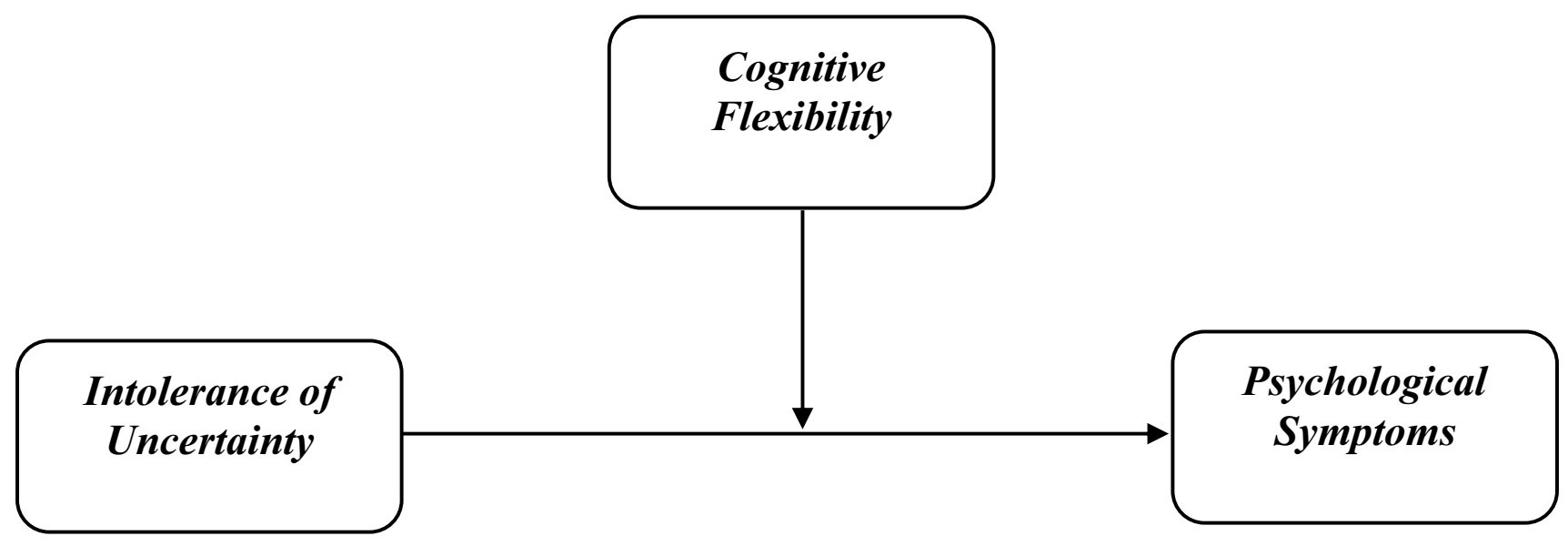

Fig. 1 The proposed model demonstrating the hypothetical associations between the study variables 


\section{Measurement Tools}

\section{Demographic Information Form}

The form was developed by the researchers to obtain information about demographics such as gender, age, marital status, education level, and perceived income level. Using the form, information about participants' occupation, whether there is any history of physical or psychological illness, how often they followed the COVID-19 news, whether they were diagnosed with COVID-19, and whether there was anyone in their environment diagnosed with COVID-19 was collected.

\section{Brief Symptom Inventory (BSI)}

Psychological symptoms were evaluated using the Brief Symptom Inventory (BSI), which is a self-report measurement tool (Derogatis, 1992). The BSI consists of 53 items in total and has a 5-point Likert rating system ranging from 0 (not-at-all) to 4 (extremely). The Cronbach's alpha values for subscales ranged from .71 to .85 in the original form (Derogatis, 1992). The Turkish form of the scale consists of five factors namely, anxiety, depression, negative self, somatization, and hostility (Şahin \& Durak, 1994). Different from the original form, one of the 12-item subscales $(15,21,22$, $24,26,34,44,48,50,51,52$, and 53) in the Turkish version was referred to as "negative self". This subscale includes items referring to the thoughts and feelings of the person regarding his/her body, personality traits, values, abilities, and the negative value he/she places on these. In the Turkish validity and reliability study, Cronbach's alpha values varied between .63 and .86 (Şahin \& Durak, 1994). In this study, the Cronbach's alpha value of the total scale was found to be .96 . Specifically, the Cronbach's alpha values were .90 for depression, .87 for anxiety, .88 for negative self, .78 for somatization, and .82 for hostility.

\section{Intolerance of Uncertainty Scale-12 (IUS-12)}

The IUS-12, which was developed by Carleton et al. (2007), was used to determine the level of intolerance to uncertainty. This 12 -item scale determines individuals' reactions to enigmatic situations, future, and uncertainty. The scale consists of two dimensions (prospective anxiety and inhibitory anxiety), has a 5-point Likert rating system with response options ranging from 1 (not at all characteristic of me) to 5 (entirely characteristic of me). The Cronbach's alpha reliability coefficient of the original scale was .91 in a non-clinical sample. In the Turkish validity and reliability study of the scale (Sarıçam et al., 2014), the Cronbach's alpha internal consistency coefficient was reported to be .88 for the total scale, .84 for prospective anxiety, and .77 for the inhibitory anxiety subdimension. Despite the reported two-factor structure, the total scale score of the IUS-12 was used in this study and the Cronbach's alpha internal consistency coefficient value was found to be .93 .

\section{Cognitive Control and Flexibility Questionnaire (CCFQ)}

The CCFQ measures the ability of the individuals to gain control over intrusive, unwanted (negative) thoughts and emotions and to cope with a stressful situation flexibly (Gabrys et al., 2018). The 18-item scale has two dimensions, namely, the cognitive control over emotion dimension and the appraisal and coping flexibility dimension and both consist of 9 items. The responses to the scale with a 7-point Likert rating system vary between 1 (strongly disagree) and 7 (strongly agree). In the original study, the Cronbach's Alpha reliability coefficients were reported to be .89 and .93 for the appraisal and coping flexibility factors and .90 for coping flexibility. The Cronbach's alpha coefficients that were calculated for the total scale and subscales in the second and third stages of the Turkish adaptation study (Demirtas, 2019) ranged from .85 to .91 . Since the aim of this study was to measure the $\mathrm{CF}$ variable, the 9-item appraisal and coping flexibility subscale was used. The Cronbach's alpha value was found to be .91 in this study.

\section{Data Collection Process}

Within the scope of this research, approval was obtained from the Turkish Ministry of Health Scientific Research Platform and Hasan Kalyoncu University Ethics Committee before the onset of data collection (grant number: -804.01E.2006060003). Upon receiving the necessary approvals, data were collected online between 1 July 2020 and 2 December 2020. The participants joined the study using an online link, and the link was delivered via social media and e-mail addresses. Before the filling out of the questionnaires, an informed consent form was presented to the participants, which discloses that their responses will be kept confidential and can only be accessed by the research team. To avoid the order effect, an online platform was programmed to present the scales and answer options in random order. Finally, the participants were asked to fill out the questionnaires. We predicted that the completion of the study by the participants would take about an average of $20 \mathrm{~min}$.

\section{Statistical Analyses}

The analysis of the data was performed using the Statistical Package for the Social Sciences version 25 (IBM SPSS 25.0) and PROCESS macro version 3.5. First, the Pearson's product-moment correlation coefficients were calculated to evaluate the relationship between research and control variables. Secondly, the moderation analysis with PROCESS 
macro version 3.5 was applied to evaluate the moderator role of $\mathrm{CF}$ while controlling the socio-demographic variables in the relationship between IU and psychological symptoms. No outliers were removed before the analyses.

\section{Results}

\section{Descriptive Statistics}

Table 1 shows the descriptive statistics (mean, standard deviation, skewness, and kurtosis) and internal consistency coefficients of the variables. The skewness values varied between -.02 and 1.13 while kurtosis values varied between -.43 and 1.22 . Thus, grounded on the criteria that skewness and kurtosis values should be equal to or greater than 2 , we deemed that all study variables had a relatively normal distribution. Moreover, the Cronbach's alpha coefficients were calculated, revealing that all scales had considerably strong internal consistency coefficients (ranging from .91 to .96).

\section{Correlational Analysis}

Table 1 shows the descriptive statistics and correlations between the demographic variables (age, gender, educational level, marital status, chronic illness, and psychiatric disorder), IU, CF, and psychological symptoms. The total BSI score was significantly associated with all variables. In particular, the total BSI score was negatively correlated with $\mathrm{CF}$ $(\mathrm{r}=-.27, \mathrm{p}<.01)$, and positively correlated with $\mathrm{IU}(\mathrm{r}=.49$, $\mathrm{p}<.01)$. The IU was positively correlated with the BSI subscales: anxiety $(\mathrm{r}=.47, \mathrm{p}<.01)$, depression $(\mathrm{r}=.46, \mathrm{p}<.01)$, negative self $(\mathrm{r}=.47, \mathrm{p}<.01)$, somatization $(\mathrm{r}=.34, \mathrm{p}<.01)$, and hostility $(\mathrm{r}=.45, \mathrm{p}<.01)$. Diversely, $\mathrm{CF}$ was found to be negatively correlated with anxiety $(\mathrm{r}=-.26, \mathrm{p}<.01)$, depression $(\mathrm{r}=-.27, \mathrm{p}<.01)$, negative self $(\mathrm{r}=-.26, \mathrm{p}<.01)$, somatization $(\mathrm{r}=-.17, \mathrm{p}<.01)$, and hostility $(\mathrm{r}=-.24, \mathrm{p}<.01)$. Finally, the IU and CF were negatively correlated with each other $(\mathrm{r}=-.13, \mathrm{p}<.01)$.

\section{Moderating Role of Cognitive Flexibility}

As a resampling method, bootstrapping was used to examine the role of the moderator variable. For that purpose, Hayes' PROCESS macro was used to determine whether CF had a moderating role in the association between IU and psychological symptoms. Accordingly, the moderator effect was investigated using Model 1 from the PROCESS models and the effects of the demographic variables (age, gender, educational level, marital status, chronic illness, and psychiatric disorder) were controlled. Unstandardized beta (B) coefficients were calculated using the bootstrapping technique, 5000 resamplings, and a confidence interval of $90 \%$.
The absence of a value of zero in the confidence interval indicated that the effects in the model were significant. Figure 2 depicts the proposed model showing the relationships between the variables.

The direct effect of the IU on the psychological symptom level was significant $\left(b_{1}=1.91, p<.01 ; 90 \%\right.$ $\mathrm{BCCI}=1.51-2.30$ ); however, the direct effect of CF on the total symptom level was not significant $\left(b_{2}=-.12\right.$, $p>.05 ; 90 \% \mathrm{BCCI}=-.45-.20)$. Importantly, the interaction between the IU and $\mathrm{CF}$ was significant $\left(b_{3}=.01, p<.05\right.$, $90 \% \mathrm{BCCI}=-.02--.002$ ). The results revealed that the level of psychological symptoms increased as the IU increased; however, the relationship between IU and psychological symptom level varied depending on the $\mathrm{CF}$ levels of the individuals. The association between the IU and psychological symptoms was significantly weaker in the high-CF condition when compared to the low-CF condition (see Fig. 3). The highest symptom level was observed in the high-IU and lowCF condition. Collectively, the moderation analysis revealed that the model was significant $(F(9,2994)=214.19, p<.01)$ and explained $39 \%$ of the variance in the psychological symptom level. Table 2 shows the results of the moderation analyses.

\section{Discussion}

The present study aimed to explore whether IU and CF have potential roles as vulnerability or protective factors in the development of psychological symptoms during the COVID-19 pandemic and, more importantly, to investigate the interaction of these cognitive factors and their associations with psychological symptoms. Although both IU and $\mathrm{CF}$ have been separately associated with various psychological disorders, the effect of their specific interaction would be important for understanding why some individuals are more prone to develop psychological symptoms despite the same uncertain and threatening conditions are true for the rest of the population as well during the COVID-19 pandemic. In line with the expectations, the results of the study revealed that psychological symptoms were positively associated with IU and negatively associated with CF. Moreover, the association between IU and psychological symptoms was moderated by $\mathrm{CF}$ after controlling for certain demographic variables.

Previous research has shown a strong association between IU and psychological symptomatology. IU was determined as a transdiagnostic maintenance factor for a variety of psychological disorders (McEvoy \& Erceg-Hurn, 2016; McEvoy \& Mahoney, 2012). The COVID-19 pandemic has brought along different uncertainties such as the lack of information about the nature of the virus, unique and changing prognosis of the disease, unknown duration of the 


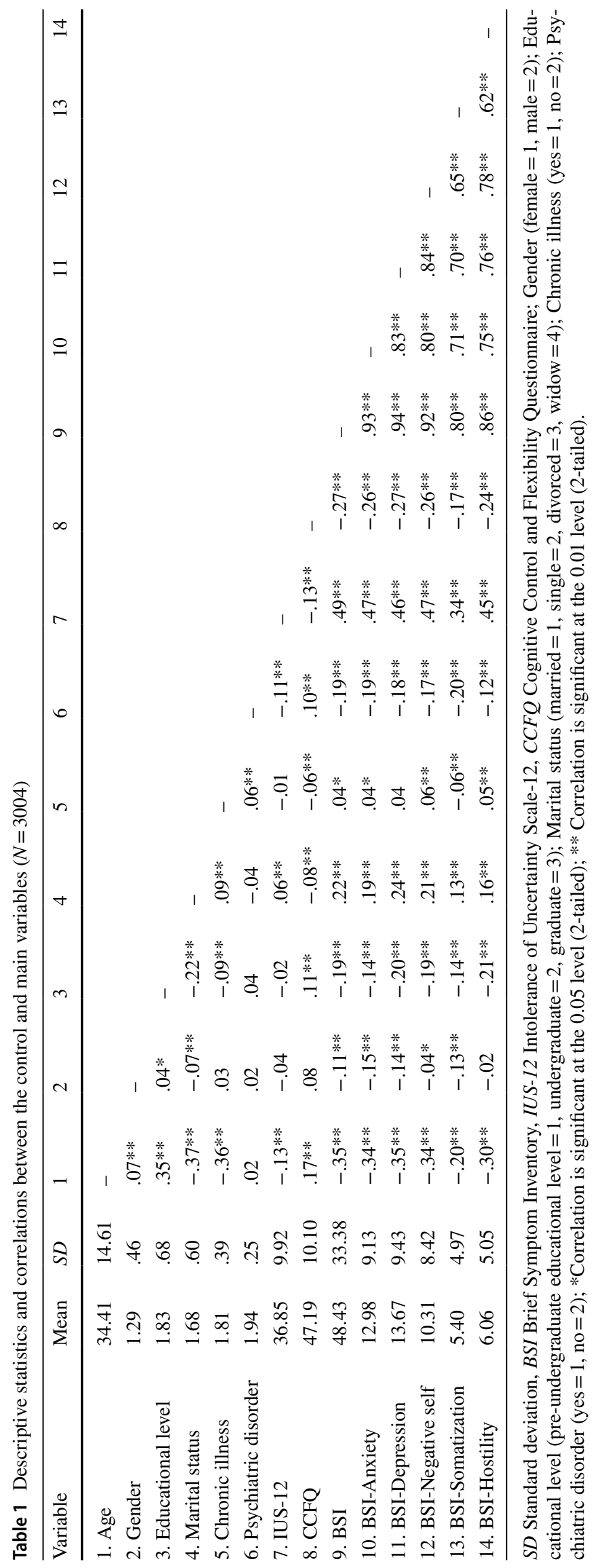




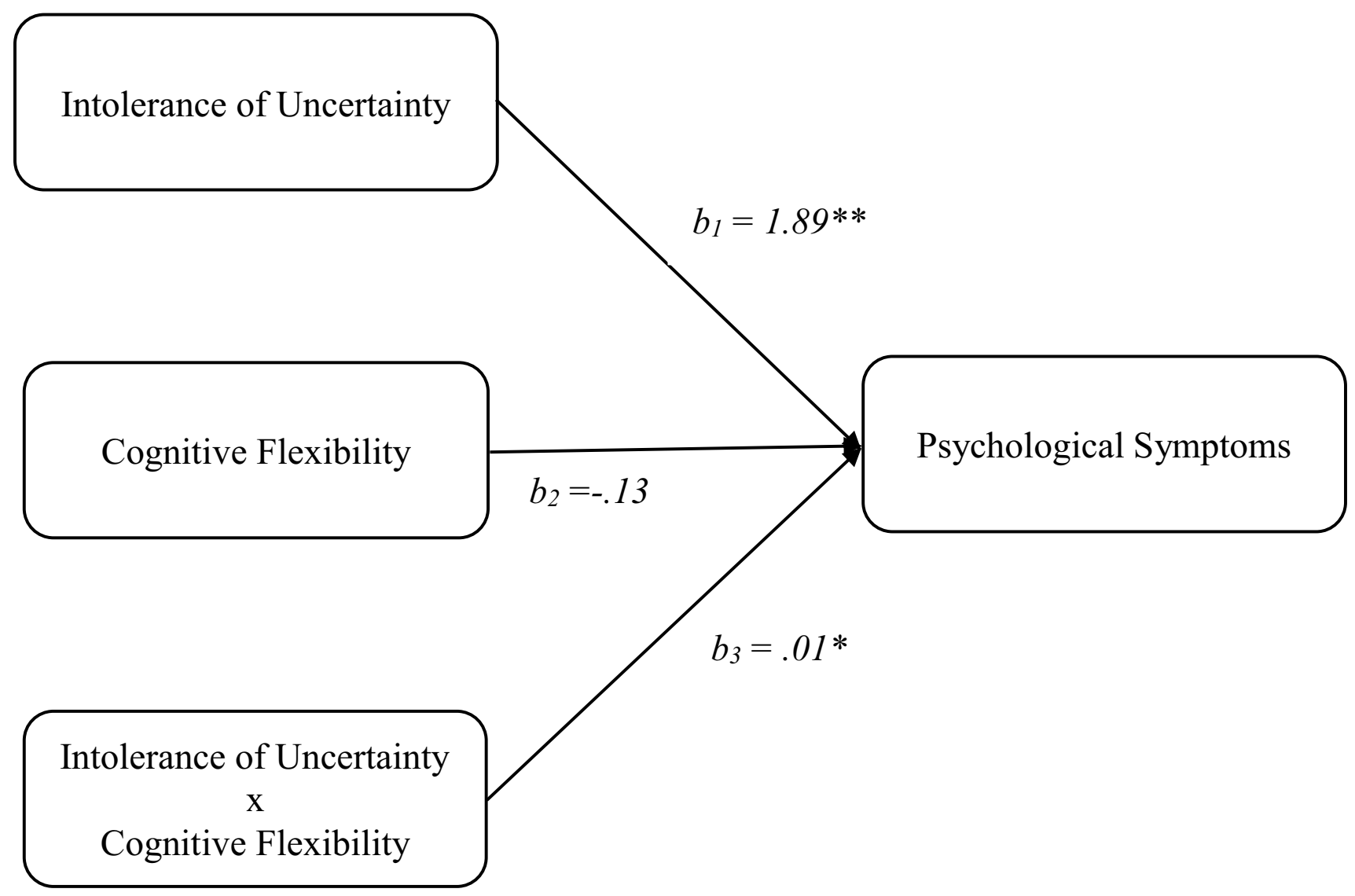

Fig. 2 Moderating role of cognitive flexibility in the relationship between the intolerance of uncertainty and psychological symptoms. ${ }^{*} p<.05$, $* * p<.01$

restrictions and social isolation practices, economic shortcomings, and many more conditions related to the pandemic. People who are already more intolerant of uncertainty experienced more psychological symptoms as they have continuously faced ambiguous circumstances during the pandemic (Parlapani et al., 2020; Satici et al., 2020; Schlegl et al., 2020; Smith et al., 2020; Voitsidis et al., 2020b). Since IU also includes beliefs about the dangerousness of uncertainty, people with high IU may react to uncertainty with avoidance or detrimental impairments in functioning such as rigidity in thoughts and behaviors, rumination, dysfunctional coping strategies, and preoccupation with uncertain events (i.e., the pandemic) (Dennis \& Vander Wal, 2010; Dugas et al., 2004; Presti et al., 2020). With this respect, the findings of the present study indicated that the IU has a direct effect on psychological symptoms and is a cognitive risk factor that is associated with psychological symptoms during the COVID-19 pandemic.

Similarly, the results supported the proposed association between the increased levels of psychological symptoms and the decreased levels of CF. However, unexpectedly; the direct effect of CF on psychological symptoms was not statistically significant according to the moderation analysis.
This finding conflicts with the literature. CF includes the ability to reappraise situations and change their interpretation, thus enabling better coping. Individuals with low $\mathrm{CF}$ engage less in reappraising, thus failing to find ways to cope with adverse situations and becoming more prone to develop psychological symptoms (Gabrys et al., 2018). CF has been associated with psychological disorders such as depression and anxiety (Dennis \& Vander Wal, 2010; Gabrys et al., 2018). From a cognitive perspective, as a part of executive functioning skills, cognitive flexibility is an ability that allows giving flexible responses to environmental contingencies (Rende, 2000). The adaptation to the restrictive and isolating conditions of COVID-19 requires cognitive reappraisal and restructuring. Therefore, the adaptation to this new context can be especially hard for individuals with lower $\mathrm{CF}$ since cognitive flexibility provides adaptive solutions to changing conditions and demands.

The results of the moderation analysis were substantial in that they revealed $\mathrm{CF}$ to be a moderator that affects the strength of the relationship between IU and psychological symptoms as being high in CF dampens the effect of IU on psychological symptoms, despite the results indicating its direct effect to be statistically not significant. Consistent 


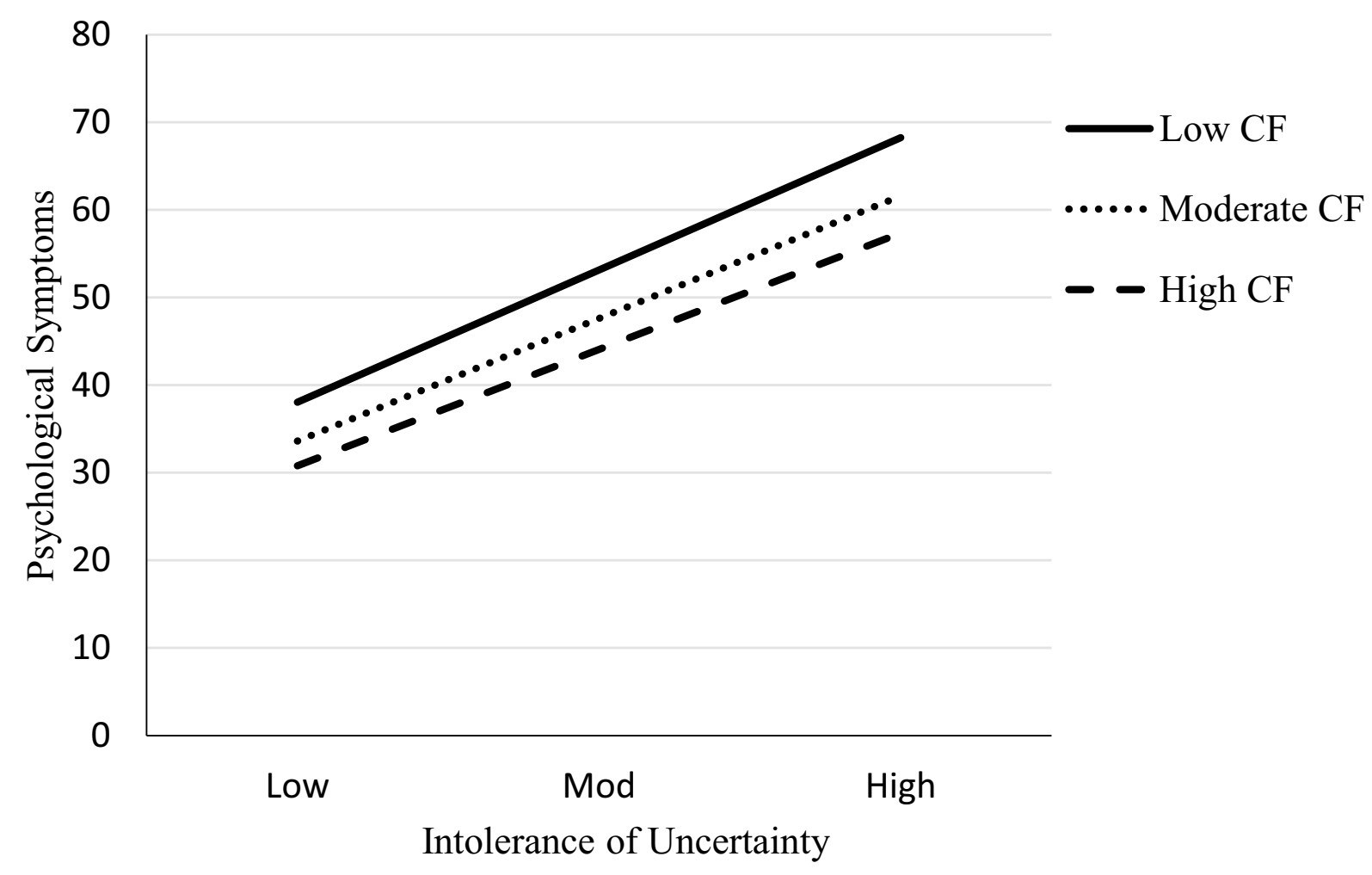

Fig. 3 Slope analysis for the interaction effect between the intolerance of uncertainty and cognitive flexibility on psychological symptoms. CF Cognitive flexibility

Table 2 Unstandardized beta coefficients and $90 \%$ bias corrected confidence intervals

\begin{tabular}{llllllll}
\hline Consequent & Antecedent & Coeff & SE & $t$ & $p$ & CI Low & CI High \\
\hline Psychological symptom & Age & -.53 & .04 & -13.44 & .00 & -.61 & -.46 \\
& Gender & -4.01 & 1.04 & -3.87 & .00 & -6.04 & -1.97 \\
& Educational level & -3.44 & .76 & -4.56 & .00 & -4.92 & -1.96 \\
& Marital status & 4.04 & .86 & 4.71 & .00 & 2.36 & 5.73 \\
& Chronic illness & -5.11 & 1.32 & -3.87 & .00 & -7.70 & -2.52 \\
& Psychiatric disorder & -15.24 & 1.97 & -7.72 & .00 & -19.11 & -11.37 \\
& Intolerance of uncertainty & 1.89 & .20 & 9.36 & .00 & 1.50 & 2.29 \\
& Cognitive flexibility & -.13 & .16 & -.79 & .43 & -.45 & .19 \\
& Intolerance of uncertainty & -.01 & .004 & -2.42 & .02 & -.02 & -.002 \\
& x Cognitive flexibility & & & & & & \\
$R^{2}$ & & .39 & & & & & \\
\hline
\end{tabular}

Coeff Unstandardized beta coefficient, SE Standard error, CI Confidence interval. with the literature (Bacaro et al., 2020; Dawson \& Golijani-Moghaddam, 2020; Demirtas \& Yildiz, 2019; Dennis \& Vander Wal, 2010; Lieberman et al., 2016; Pakenham et al., 2020), the association between IU and psychological symptoms is weaker in a person with high CF compared to that in a person with low CF. As a construct, CF is defined as shifting thoughts and increasing behavioral responses for better adaptation. Therefore, CF can serve as a protective factor for adverse behaviors, thoughts, and emotions resulting from high IU (Presti et al., 2020). While high IU was associated with negative reactions to uncertainty such as worrying, anxiety, and avoidance, high CF was associated with cognitive restructuring, adjustment to uncertainty, and reduced levels of psychological symptoms. The negative 
association between IU and CF was supported by previous research as well. One study on people with panic disorder showed the mediating role of $\mathrm{CF}$ in the relationship between IU and defensive responding (i.e., startle response). Despite not being in danger, people with high IU could not shift their focus from past threats due to low $\mathrm{CF}$ and displayed increased levels of the startle response (Lieberman et al., 2016). Another study demonstrated the serial mediation of $\mathrm{CF}$ and IU in the relationship between hopelessness and perceived stress. People who had higher hopelessness also had lower CF, which, in turn, exacerbated IU and stress (Demirtas \& Yildiz, 2019). According to evidence from this and previous research, although high IU creates difficulty in adapting to ambiguity, high CF can enable shifting away from an IU-related biased way of thinking. In other words, the findings of the moderation model suggested that having high $\mathrm{CF}$ as a possible protective factor can compensate for the adverse impact of IU on psychological well-being by enabling cognitive restructuring and more effective coping.

\section{Limitations and Strengths of the Study}

Certain methodological limitations should be considered when interpreting the results. First, all variables were evaluated using self-report questionnaires and, thus, can be subject to social desirability. Furthermore, the participation in this study was on a voluntary basis, which may have led to self-selection bias. Moreover, snowball sampling was used to reach a larger number of participants, but this sampling strategy may not be the best option to represent the general population as it is not based on randomization. Similarly, students constitute a large portion of the participants, constituting a problem for representativeness as well.

The present study has a high female-to-male ratio. Specifically, $71.2 \%$ of the participants were female and $28.4 \%$ were male. This might negatively affect the generalizability of the results to the population. In the literature, women have been reported to be at a greater risk to experience psychological symptoms and intolerance of uncertainty during the pandemic (Liu et al., 2020; Mazza et al., 2020; Parlapani et al., 2020). In terms of cognitive flexibility, previous studies chose to control gender as a variable and did not mention any group differences (Dawson \& Golijani-Moghaddam, 2020). In the present study, we did not aim to examine the group differences according to gender either. We controlled gender as a variable when testing the hypothesized model. Still, the unequal female-to-male ratio should be considered when interpreting the results.

As another limitation of the study, data were collected cross-sectionally. Thus, the long-term effects of COVID-19 on psychological symptoms may not be inferred using the current results. In addition, the data collection period was expanded over 6 months, which is a relatively large period during the pandemic and may lead to a confounding effect in the interpretation of the results. Finally, the moderation model explained $39 \%$ of the variance in the psychological symptoms, which clearly signifies the existence of other variables that can also influence the development of psychological symptoms.

Notwithstanding its limitations, this research presented valuable information about the current status of the psychological symptoms in Turkey and the importance of IU and $\mathrm{CF}$ during the pandemic. The present study had a relatively large samples size with 3004 participants. Therefore, the results were considered to have high validity and generalizability. Importantly, the present study investigated two cognitive variables (IU and $\mathrm{CF}$ ) that have an important role in the development of psychological symptoms even after controlling for socio-demographic variables that induce increased risk. This highlights the potential benefits of using IU and $\mathrm{CF}$ in the clinical setting.

\section{Clinical Implications and Suggestions for Interventions}

The COVID-19 pandemic can be defined as an unprecedented period. Economic hardship, unemployment, changes in the educational system, imposed restrictions and cautionary actions, uneasiness about possible shortages in supplies, and many more are part of the aftermath of this period (Moreno et al., 2020; Stanton et al., 2020; Voitsidis et al., 2020b). Individuals around the world had to adapt to unusual consequences that were accompanied by the feeling of uncertainty and ambiguity, which also caused increases in stress and anxiety (Smith et al., 2020; Voitsidis et al., 2020b). These responses were plausible to some degree; however, studies have reported more negative mental health outcomes for people with high IU and low CF (Satici et al., 2020; Schlegl et al., 2020).

Prior research recommended specifically targeting IU and CF to alleviate the severity of psychological symptoms (McEvoy \& Erceg-Hurn, 2016; McEvoy \& Mahoney, 2012; Presti et al., 2020). Emphasizing both constructs in psychological interventions and providing information to individuals to promote self-guided interventions are substantial in preserving mental health. The application of CF in therapy corresponds to the intervention methods such as cognitive restructuring and alternative thought generation in cognitive-behavioral therapy (Dennis \& Vander Wal, 2010; Gabrys et al., 2018). Acceptance and Commitment Therapy is also an approach that targets psychological flexibility, a construct overlapping with $\mathrm{CF}$, which was previously shown to be useful in alleviating psychological symptoms during the COVID-19 pandemic (Smith et al., 2020). Similarly, IU has been incorporated into therapy by evaluating and challenging dysfunctional beliefs, thoughts, emotions, and 
coping styles that are related to uncertainty (McEvoy \& Erceg-Hurn, 2016). The results of the present study further emphasize the need to integrate both constructs in the interventions targeting COVID 19 pandemic related distress. The main aim of these interventions should be the assessment of thoughts and emotions regarding the pandemic and developing a better understanding of the reactions to uncertainty and possible cognitive rigidity stemming from anxiety or fear. Subsequently, the interventions should focus on challenging any negative interpretations, generating alternative thoughts, and promoting the existing functional coping mechanisms. These strategies can help tolerate the uncertainties through elevated CF and alleviate psychological symptoms (McEvoy \& Erceg-Hurn, 2016; Presti et al., 2020; Smith et al., 2020).

\section{Further Research}

Future studies should focus on both short-term and longterm psychological outcomes of the COVID-19 pandemic, including various factors affecting psychological wellbeing. It would be helpful to investigate the effects of psychological interventions that target IU and CF and to assess their effectiveness in decreasing psychological symptoms. Moreover, the effect of IU and CF can be investigated using a longitudinal research design to better understand the importance of the results. In this study, we have examined only the moderating role of CF in the association between IU and psychological symptoms during COVID-19 pandemic. We strongly encourage the future studies to test a more complex model including the factors that would interact with the intolerance of uncertainty in the context of COVID-19 such as fear of infection, and infecting others, lockdowns, uncertainty about accessing to healthcare, the waves of the epidemic and new viral strains, loss of job, income, and education.

\section{Conclusion}

The current study investigated the moderating effect of cognitive flexibility in the relationship between intolerance of uncertainty and psychological symptoms in the context of the COVID-19 pandemic. The results can increase our understanding of why some individuals are more susceptible to experience psychological symptoms in such uncertain and changing conditions that the pandemic entails. In line with our expectations, the results showed that IU is positively correlated with psychological symptoms and seems to be a potential risk factor for developing psychological symptoms. On the other hand, CF is negatively correlated with psychological symptoms and serves a protective function. More importantly, the association between IU and psychological symptoms was moderated by $\mathrm{CF}$ after controlling for various demographic variables. This finding suggested that having high CF (being able to shift away from negative thoughts) could compensate for the adverse impact of IU (thinking that uncertainty is threatening) on psychological well-being. Psychological interventions that target the improvement of $\mathrm{CF}$ and elimination of IU should be utilized to reduce psychological symptoms. Strategies such as thought challenging, cognitive restructuring, and promoting different coping mechanisms can be used in personal or group intervention settings.

Author Contributions Mujgan Inozu, B. Göktürk Gök, Duygu Tuzun, and A. Bikem Haciomeroglu designed the study. B. Göktürk Gök and Duygu Tuzun collected, analyzed the data, and wrote the introduction, methodology, results, discussion, and conclusion sections of the manuscript. A. Bikem Haciomeroglu and Mujgan Inozu edited the manuscript and finalized it.

Funding This study was funded by The Scientific and Technological Research Council of Turkey (TÜBİTAK) with grant number 120K408.

Data Availability The data used in the analysis are available from the corresponding author on to a reasonable request.

Code Availability Not applicable.

\section{Declarations}

Conflict of Interest We have no known conflict of interest to disclose.

Ethics Approval This study was approved by the Hasan Kalyoncu University Ethics Committee (Ethics approval number: -804.01E.2006060003).

Informed Consent Online consent form was obtained from the participants after all necessary explanations about the study were given.

\section{References}

Bacaro, V., Chiabudini, M., Buonanno, C., De Bartolo, P., Riemann, D., Mancini, F., \& Baglioni, C. (2020). Insomnia in the Italian population during Covid-19 outbreak: A snapshot on one major risk factor for depression and anxiety. Frontiers in Psychiatry, 11, 579107. https://doi.org/10.3389/fpsyt.2020.579107

Buhr, K., \& Dugas, M. J. (2002). The intolerance of uncertainty scale: Psychometric properties of the English version. Behavior Research and Therapy, 40(8), 931-945. https://doi.org/10.1016/ S0005-7967(01)00092-4

Cao, W., Fang, Z., Hou, G., Han, M., Xu, X., Dong, J., \& Zheng, J. (2020). The psychological impact of the COVID-19 epidemic on college students in China. Psychiatry Research, 287, 112934. https://doi.org/10.1016/j.psychres.2020.112934

Carleton, R. N., Norton, M. P. J., \& Asmundson, G. J. (2007). Fearing the unknown: A short version of the intolerance of uncertainty scale. Journal of Anxiety Disorders, 21(1), 105-117. https://doi. org/10.1016/j.janxdis.2006.03.014

Cheng, C., Lau, H.-P. B., \& Chan, M.-P. S. (2014). Coping flexibility and psychological adjustment to stressful life changes: A 
meta-analytic review. Psychological Bulletin, 140(6), 1582-1607. https://doi.org/10.1037/a0037913

Dawson, D. L., \& Golijani-Moghaddam, N. (2020). COVID-19: Psychological flexibility, coping, mental health, and wellbeing in the UK during the pandemic. Journal of Contextual Behavioral Science, 17, 126-134. https://doi.org/10.1016/j.jcbs.2020.07.010

Demirtaş, A. S. (2019). Stresli durumlarda bilişsel kontrol ve bilişsel esneklik: Bir ölçek uyarlama çalışması. Psikoloji Çalışmaları, 39(2), 345-368. https://doi.org/10.26650/SP2019-0028

Demirtas, A. S., \& Yildiz, B. (2019). Hopelessness and perceived stress: The mediating role of cognitive flexibility and intolerance of uncertainty. Dusunen Adam the Journal of Psychiatry and Neurological Sciences, 32(3), 259. https://doi.org/10.14744/DAJPNS. 2019.00035

Dennis, J. P., \& Vander Wal, J. S. (2010). The cognitive flexibility inventory: Instrument development and estimates of reliability and validity. Cognitive Therapy and Research, 34(3), 241-253. https://doi.org/10.1007/s10608-009-9276-4

Derogatis, L. R. (1992). The brief symptom inventory (BSI): Administration, scoring, and procedures manual-II (2nd ed.). National Computer Systems.

Diamond, A. (2013). Executive functions. Annual Review of Psychology, 64, 135-168. https://doi.org/10.1146/annur ev-psych-113011-143750

Dugas, M. J., \& Ladouceur, R. (2000). Treatment of GAD: Targeting intolerance of uncertainty in two types of worry. Behavior Modification, 24(5), 635-657. https://doi.org/10.1177/01454 45500245002

Dugas, M. J., Schwartz, A., \& Francis, K. (2004). Brief report: Intolerance of uncertainty, worry, and depression. Cognitive Therapy and Research, 28(6), 835-842. https://doi.org/10.1007/ s10608-004-0669-0

Fernández, R. S., Crivelli, L., Guimet, N. M., Allegri, R. F., \& Pedreira, M. E. (2020). Psychological distress associated with COVID-19 quarantine: Latent profile analysis, outcome prediction and mediation analysis. Journal of Affective Disorders, 277, 75-84. https:// doi.org/10.1016/j.jad.2020.07.133

Gabrys, R. L., Tabri, N., Anisman, H., \& Matheson, K. (2018). Cognitive control and flexibility in the context of stress and depressive symptoms: The cognitive control and flexibility questionnaire. Frontiers in Psychology, 9, 2219. https://doi.org/10.3389/fpsyg. 2018.02219

Gorka, S. M., Lieberman, L., Nelson, B. D., Sarapas, C., \& Shankman, S. A. (2014). Aversive responding to safety signals in panic disorder: The moderating role of intolerance of uncertainty. Journal of Anxiety Disorders, 28(7), 731-736. https://doi.org/10.1016/j. janxdis.2014.06.004

Huang, Y., \& Zhao, N. (2021). Mental health burden for the public affected by the COVID-19 outbreak in China: Who will be the high-risk group? Psychology, Health \& Medicine, 26(1), 23-34. https://doi.org/10.1080/13548506.2020.1754438

Li, L. Z., \& Wang, S. (2020). Prevalence and predictors of general psychiatric disorders and loneliness during COVID-19 in the United Kingdom. Psychiatry Research, 291, 113267. https://doi.org/10. 1016/j.psychres.2020.113267

Lieberman, L., Gorka, S. M., Sarapas, C., \& Shankman, S. A. (2016). Cognitive flexibility mediates the relation between intolerance of uncertainty and safety signal responding in those with panic disorder. Cognition and Emotion, 30(8), 1495-1503. https://doi. org/10.1080/02699931.2015.1067189

Lind, C., \& Boschen, M. J. (2009). Intolerance of uncertainty mediates the relationship between responsibility beliefs and compulsive checking. Journal of Anxiety Disorders, 23(8), 1047-1052. https:// doi.org/10.1016/j.janxdis.2009.07.005

Liu, N., Zhang, F., Wei, C., Jia, Y., Shang, Z., Sun, L., et al. (2020). Prevalence and predictors of PTSS during COVID-19 outbreak in China hardest-hit areas: Gender differences matter. Psychiatry Research, 287, 112921. https://doi.org/10.1016/j.psychres. 2020.112921

Mazza, C., Ricci, E., Biondi, S., Colasanti, M., Ferracuti, S., Napoli, C., \& Roma, P. (2020). A nationwide survey of psychological distress among Italian people during the COVID-19 pandemic: Immediate psychological responses and associated factors. International Journal of Environmental Research and Public Health, 17(9), 3165. https://doi.org/10.3390/ijerph17093165

McEvoy, P. M., \& Mahoney, A. E. (2012). To be sure, to be sure: Intolerance of uncertainty mediates symptoms of various anxiety disorders and depression. Behavior Therapy, 43(3), 533545. https://doi.org/10.1016/j.beth.2011.02.007

McEvoy, P. M., \& Erceg-Hurn, D. M. (2016). The search for universal transdiagnostic and trans-therapy change processes: Evidence for intolerance of uncertainty. Journal of Anxiety Disorders, 41, 96-107. https://doi.org/10.1016/j.janxdis.2016.02.002

Moreno, C., Wykes, T., Galderisi, S., Nordentoft, M., Crossley, N., Jones, N., et al. (2020). How mental health care should change as a consequence of the COVID-19 pandemic. The Lancet Psychiatry. https://doi.org/10.1016/S2215-0366(20)30307-2

Pakenham, K. I., Landi, G., Boccolini, G., Furlani, A., Grandi, S., \& Tossani, E. (2020). The moderating roles of psychological flexibility and inflexibility on the mental health impacts of COVID-19 pandemic and lockdown in Italy. Journal of Contextual Behavioral Science, 17, 109-118. https://doi.org/10. 1016/j.jcbs.2020.07.003

Parlapani, E., Holeva, V., Nikopoulou, V. A., Sereslis, K., Athanasiadou, M., Godosidis, A., et al. (2020). Intolerance of uncertainty and loneliness in older adults during the COVID-19 pandemic. Frontiers in Psychiatry, 11, 842. https://doi.org/10.3389/fpsyt. 2020.00842

Petzold, M. B., Bendau, A., Plag, J., Pyrkosch, L., Mascarell Maricic, L., Betzler, F., et al. (2020). Risk, resilience, psychological distress, and anxiety at the beginning of the COVID-19 pandemic in Germany. Brain and Behavior, 10(9), e01745. https://doi.org/ 10.1002/brb3.1745

Presti, G., Mchugh, L., Gloster, A., Karekla, M., \& Hayes, S. C. (2020). The dynamics of fear at the time of covid-19: A contextual behavioral science perspective. Clinical Neuropsychiatry, 17(2), 65-71. https://doi.org/10.36131/CN20200206

Rende, B. (2000). Cognitive flexibility: Theory, assessment and treatment. Seminars in Speech and Language, 21(2). https:// doi.org/10.1055/s-2000-7560

Sarıçam, H., Erguvan, F. M., Akın, A., \& Akça, M. Ş. (2014). The Turkish short version of the intolerance of uncertainty (IUS-12) scale: The study of validity and reliability. Route Educational and Social Science Journal, 1(3), 148-157.

Satici, B., Saricali, M., Satici, S. A., \& Griffiths, M. D. (2020). Intolerance of uncertainty and mental wellbeing: Serial mediation by rumination and fear of COVID-19. International Journal of Mental Health and Addiction, 15, 1-12. https://doi.org/10.1007/ s11469-020-00305-0

Schlegl, S., Maier, J., Meule, A., \& Voderholzer, U. (2020). Eating disorders in times of the COVID-19 pandemic-Results from an online survey of patients with anorexia nervosa. International Journal of Eating Disorders, 53(11), 1791-1800. https://doi. org/10.1002/eat.23374

Smith, B. M., Twohy, A. J., \& Smith, G. S. (2020). Psychological inflexibility and intolerance of uncertainty moderate the relationship between social isolation and mental health outcomes during COVID-19. Journal of Contextual Behavioral Science, 18, 162-174. https://doi.org/10.1016/j.jcbs.2020.09.005

Stanton, R., To, Q. G, Khalesi, S., Williams, S. L., Alley, S. J., Thwaite, T. L., et al. (2020). Depression, anxiety and stress during COVID-19: Associations with changes in physical activity, 
sleep, tobacco and alcohol use in Australian adults. International Journal of Environmental Research and Public Health, 17(11), 4065. https://doi.org/10.3390/ijerph17114065

Şahin, N. H., \& Durak, A. (1994). Kısa semptom envanteri: Türk gençleri için uyarlanması. Türk Psikoloji Dergisi, 9(31), 44-56.

Tian, F., Li, H., Tian, S., Yang, J., Shao, J., \& Tian, C. (2020). Psychological symptoms of ordinary Chinese citizens based on SCL-90 during the level I emergency response to COVID-19. Psychiatry Research, 288, 112992. https://doi.org/10.1016/j.psychres.2020. 112992

Varshney, M., Parel, J. T., Raizada, N., \& Sarin, S. K. (2020). Initial psychological impact of COVID-19 and its correlates in Indian community: An online (FEEL-COVID) survey. PLoS One, 15(5), e0233874. https://doi.org/10.1371/journal.pone.0233874

Voitsidis, P., Gliatas, I., Bairachtari, V., Papadopoulou, K., Papageorgiou, G., Parlapani, E., et al. (2020a). Insomnia during the COVID-19 pandemic in a Greek population. Psychiatry Research, 289, 113076. https://doi.org/10.1016/j.psychres.2020.113076

Voitsidis, P., Nikopoulou, V. A., Holeva, V., Parlapani, E., Sereslis, K., Tsipropoulou, V., et al. (2020b). The mediating role of fear of COVID-19 in the relationship between intolerance of uncertainty and depression. Psychology and Psychotherapy: Theory, Research and Practice. https://doi.org/10.1111/papt.12315

Wang, C., Pan, R., Wan, X., Tan, Y., Xu, L., Ho, C. S., \& Ho, R. C. (2020). Immediate psychological responses and associated factors during the initial stage of the 2019 coronavirus disease (COVID19) epidemic among the general population in China. International Journal of Environmental Research and Public Health, 17(5), 1729. https://doi.org/10.3390/ijerph17051729

Whiting, D. L., Deane, F. P., Simpson, G. K., McLeod, H. J., \& Ciarrochi, J. (2017). Cognitive and psychological flexibility after a traumatic brain injury and the implications for treatment in acceptance-based therapies: A conceptual review. Neuropsychological Rehabilitation, 27(2), 263-299. https://doi.org/10.1080/09602011. 2015.1062115

WHO. (2020). WHO director-general's opening remarks at the media briefing on covid-19 - 11 MARCH 2020. Retrieved February 17, 2021, from https://www.who.int/director-general/speeches/detail/ who-director-general-s-opening-remarks-at-the-media-briefingon-covid-19\%2D\%2D-11-march-2020.

WHO. (2021). WHO COVID-19 Explorer. Retrieved October 1, 2021, from https://worldhealthorg.shinyapps.io/covid/

Xiong, J., Lipsitz, O., Nasri, F., Lui, L. M., Gill, H., Phan, L., et al. (2020). Impact of COVID-19 pandemic on mental health in the general population: A systematic review. Journal of affective disorders, 277, 55-64. https://doi.org/10.1016/j.jad.2020.08.001

Ye, Z., Yang, X., Zeng, C., Wang, Y., Shen, Z., Li, X., \& Lin, D. (2020). Resilience, social support, and coping as mediators between COVID-19-related stressful experiences and acute stress disorder among college students in China. Applied Psychology: Health and Well-Being, 12(4), 1074-1094. https://doi.org/10. 1111/aphw.12211

Yook, K., Kim, K. H., Suh, S. Y., \& Lee, K. S. (2010). Intolerance of uncertainty, worry, and rumination in major depressive disorder and generalized anxiety disorder. Journal of Anxiety Disorders, 24(6), 623-628. https://doi.org/10.1016/j.janxdis.2010.04.003

Publisher's Note Springer Nature remains neutral with regard to jurisdictional claims in published maps and institutional affiliations. 